This is an Open Access article, distributed under the terms of the Creative Commons Attribution licence (http://creativecommons.org/licenses/by/4.0/), which permits unrestricted re-use, distribution, and reproduction in any medium, provided the original work is properly cited.

doi:10.1017/jfm.2016.162

\title{
On the steady-state nearly resonant waves
}

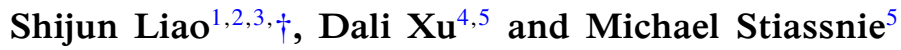 \\ ${ }^{1}$ State Key Laboratory of Ocean Engineering, Shanghai 200240, China \\ ${ }^{2}$ Collaborative Innovation Center for Advanced Ship and Deep-Sea Exploration (CISSE), \\ Shanghai 200240, China \\ ${ }^{3}$ School of Naval Architecture, Ocean and Civil Engineering, Shanghai Jiao Tong University, \\ Shanghai 200240, China \\ ${ }^{4}$ College of Ocean Science and Engineering, Shanghai Maritime University, Shanghai 201306, China \\ ${ }^{5}$ Faculty of Civil and Environmental Engineering, Technion IIT, Haifa 32000, Israel
}

(Received 5 May 2015; revised 30 January 2016; accepted 22 February 2016; first published online 30 March 2016)

The steady-state nearly resonant water waves with time-independent spectrum in deep water are obtained from the full wave equations for inviscid, incompressible gravity waves in the absence of surface tension by means of a analytic approximation approach based on the homotopy analysis method (HAM). Our strategy is to mathematically transfer the steady-state nearly resonant wave problem into the steady-state exactly resonant ones. By means of choosing a generalized auxiliary linear operator that is a little different from the linear part of the original wave equations, the small divisor, which is unavoidable for nearly resonant waves in the frame of perturbation methods, is avoided, or moved far away from low wave frequency to rather high wave frequency with physically negligible wave energy. It is found that the steady-state nearly resonant waves have nothing fundamentally different from the steady-state exactly resonant ones, from physical and numerical viewpoints. In addition, the validity of this HAM-based analytic approximation approach for the full wave equations in deep water is numerically verified by means of the Zakharov's equation. A thought experiment is discussed, which suggests that the essence of the so-called 'wave resonance' should be reconsidered carefully from both of physical and mathematical viewpoints.

Key words: surface gravity waves, waves/free-surface flows

\section{Introduction}

About a half century ago, using a perturbation method (at the third order) that has been widely applied in fluid mechanics for a long time, Phillips (1960) gave the criterion of wave resonance

$$
\boldsymbol{k}_{1}+\boldsymbol{k}_{2}=\boldsymbol{k}_{3}+\boldsymbol{k}_{4}, \quad \omega_{1}+\omega_{2}=\omega_{3}+\omega_{4},
$$

$†$ Email address for correspondence: sjliao@sjtu.edu.cn 
where $\boldsymbol{k}_{i}$ is wavenumber and $\omega_{i}=\sqrt{g k_{i}}$ (with the gravity acceleration $g$ and the definition $k_{i}=\left|\boldsymbol{k}_{i}\right|$ ) is the linear frequency. In addition, Phillips (1960) revealed that amplitude of resonant wave component grows linearly with time, if it is zero initially. Thereafter, the exactly and nearly resonant waves have been widely studied and applied (Benney 1962; Longuet-Higgins 1962; Bretherton 1964; Longuet-Higgins \& Smith 1966; Stiassnie \& Shemer 1984; Hammack \& Henderson 1993; Mei, Stiassnie \& Yue 2005; Onorato et al. 2009). In particular, Benney (1962) established the evolution equations of wave-mode amplitudes, and demonstrated the time-dependent periodic exchange of wave energy for exactly resonant waves. Benney (1962) mentioned that 'if all three modes are initially present then the energy distribution will vary with time' and thus 'no stable equilibrium amplitudes can be expected as the system is conservative.' To the best of our knowledge, the so-called steady-state resonant waves (with time-independent spectrum) have not been found analytically at a order higher than three from the full water wave equations, because perturbation results contain secular terms when Phillips' criterion is satisfied so that 'the perturbation theory breaks down due to singularities in the transfer functions', as pointed out by Madsen \& Fuhrman (2012).

The nonlinear interactions of steady-state periodic travelling water waves were investigated by many researchers, but mostly without considering wave resonance. Concus (1962) analytically investigated the standing capillary-gravity waves of finite amplitude by means of perturbation methods, but without considering the exact or near resonance of the wave. Hammack, Scheffner \& Segur (1989), Hammack, Henderson \& Segur (2005) experimentally studied in a wave tank the interaction of two families of periodic travelling waves in shallow water, and observed pictures where symmetric and asymmetric hexagons were formed by wave crests. They also analytically reproduced such solutions using the Kadomtsev-Petviashvili (KP) equation which models this behaviour well. Note that Hammack et al. $(1989,2005)$ did not consider exact/near resonance of the wave either. In addition, Dubrovin, Flickinger \& Segur (1997) gave closed-form analytic solutions of the KP equation with three phases for travelling waves in shallow water, and found that 'almost every three-phase solution is time dependent, in every coordinate system'. In addition, Iooss \& Plotnikov $(2009,2011)$ seriously proved the existence of the symmetrical and non-symmetrical three-dimensional travelling gravity waves without exact/near resonance.

Meiron, Saffman \& Yuen (1982) numerically solved the full wave equations for steady three-dimensional symmetric gravity waves with finite amplitude on deep water, and obtained results in good agreement with the experimental observations of Su (1982) and Su et al. (1982). Note that the exact/near resonance was not considered by Su (1982), Su et al. (1982) and Meiron et al. (1982). For free surface waves with arbitrary uniform depth, Milewski \& Keller (1996) derived an isotropic pseudo-differential equation at the second order of small perturbation quantities, i.e. the ratio of wave amplitude to the wavelength and the ratio of depth to wavelength, from which one can reduce to the Benney-Luke equation, the Korteweg-de Vries $(\mathrm{KdV})$ equation, the KP equation, and to the nonlinear shallow-water equations in the appropriate limits. Using their second-order perturbation equations together with the resonance criterion given by Phillips (1960) at the third order, Milewski \& Keller (1996) numerically obtained steady-state resonant and non-resonant waves in finite water depth, although no multiple solutions were reported. Using a spectral collocation discretization together with the continuous method, Nicholls (1998) investigated travelling wave solutions to the Zakharov-Craig-Sulem (ZCS) formulation 
of the water wave problem, which is equivalent to the full water wave equations, but without considering exactly or nearly resonant waves. Based on a kind of integro-differential equations, Craig \& Nicholls (2002) numerically investigated the bifurcation of travelling surface water waves, using the fast Fourier transform and surface spectral methods proposed by Nicholls (1998). However, as pointed out by Craig \& Nicholls (2002), the three-dimensional waves (cross-waves) without surface tension exhibits the phenomenon of small divisors, which could make the behaviour of their numerical scheme very difficult to control. Nicholls \& Reitich (2006) proposed a stable high-order boundary perturbation algorithm for the numerical simulation of travelling water waves, but pointed out that the resonance 'lies outside the scope' of their method. Meiron et al. (1982) also mentioned that, 'the small-divisor problem appears if an attempt is made to calculate solutions by developing expansions in wave height', and that 'we are faced with a situation where increasing the accuracy of a calculation may lead to an increase in the number of close solutions and a decrease in the physical precision.' To the best of our knowledge, numerical methods were not successful to the full water wave equations for either exactly or nearly resonant waves, as pointed out by Meiron et al. (1982), Craig \& Nicholls (2002) and Nicholls $\&$ Reitich (2006). Neither do perturbation methods for the full wave equations at an order higher than three, as mentioned by Madsen \& Fuhrman (2012).

By means of the homotopy analysis method (HAM) (Liao 1992, 1997, 2003, 2004, 2012, 2014; Vajravelu \& Van Gorder 2013), an analytic approximation method for highly nonlinear problems, the multiple solutions of steady-state resonant waves were obtained in the form of analytic approximations from the full water wave equations, not only in deep water (Liao 2011; Liu \& Liao 2014) but also in uniform water depth (Xu et al. 2012) and over a bottom with an infinite number of periodic ripples (Xu, Lin \& Liao 2015). In particular, the multiple steady-state resonant waves were even observed experimentally (Liu et al. 2015) in a basin at State Key Laboratory of Ocean Engineering (in Shanghai, China), with excellent agreement with their theoretical predictions given by means of the HAM.

When Phillips' resonance criterion (1.1) is exactly satisfied, it is traditionally called the exact resonance. When it is not exactly but nearly satisfied, it is called the nearly resonant wave. However, it is well known that near-resonance leads to the famous 'small divisor problem' (Poincaré 1892; Yoccoz 1992), which is difficult to handle in mathematics (even numerically), as pointed out by Meiron et al. (1982), Craig \& Nicholls (2002) and Nicholls \& Reitich (2006). To the best the authors' knowledge, the multiple steady-state nearly resonant surface waves with time-independent spectrum have not been gained from the full water equations by means of perturbation methods either (Madsen \& Fuhrman 2012).

In this paper, we further applied the HAM to the full water wave equations in deep water and successfully gained such kind of steady-state nearly resonant waves with time-independent spectrum in a way rather similar to that for the exactly resonant waves (Liao 2011; Xu et al. 2012; Liu \& Liao 2014). In the frame of the HAM, by means of choosing a new linear operator that is a little different from the linear part of the original equations (here, it should be emphasized that it is impossible to do the same thing in the frame of perturbation methods), we can easily avoid the small divisor at the low wave frequency, or at least move it far away to a quite high frequency with physically negligible wave energy. From the physical and numerical viewpoints, it is found that the steady-state nearly resonant waves have nothing fundamentally different from the steady-state exactly resonant ones. These conclusions are confirmed and verified numerically by means of the Zakharov's 
equation in a similar way to the steady-state exactly resonant case (Xu et al. 2012). Therefore, the steady-state nearly resonant waves have nothing fundamentally different from the steady-state exactly resonant ones, from the viewpoints of the HAM.

In this paper, the mathematical formulae are described in $\S 2$, and results of a case study are given in $\S 3$. The concluding remarks and discussions are given in $\S 4$. The detailed analytic approximation approach based on the HAM for the full wave equation is given in appendix A and the numerical algorithm based on Zakharov's equation is briefly described in appendix B.

\section{Mathematical formulae}

Consider the nonlinear interactions of two trains of progressive gravity waves with small amplitudes in deep water. Assume that the fluid is inviscid and incompressible, the flow is irrotational and the surface tension is neglected. The coordinate system $(x, y, z)$ is set on the free surface, with $z$-axis positive vertically upwards. The velocity potential $\varphi(x, y, z, t)$ is governed by the Laplace equation

$$
\nabla^{2} \varphi=0, \quad-\infty<z<\eta(x, y, t),
$$

subject to the kinematic and dynamic conditions on the unknown free surface $z=\eta(x, y, t)$ :

$$
\begin{gathered}
\frac{\partial^{2} \varphi}{\partial t^{2}}+g \frac{\partial \varphi}{\partial z}+\frac{\partial|\nabla \varphi|^{2}}{\partial t}+\nabla \varphi \cdot \nabla\left(\frac{1}{2}|\nabla \varphi|^{2}\right)=0, \\
g \eta+\frac{\partial \varphi}{\partial t}+\frac{1}{2}|\nabla \varphi|^{2}=0
\end{gathered}
$$

respectively, and the impermeable condition at the bottom:

$$
\lim _{z \rightarrow-\infty} \frac{\partial \varphi}{\partial z}=0,
$$

where $\eta(x, y, t)$ denotes the wave elevation, $t$ denotes the time and

$$
\nabla=\boldsymbol{i} \frac{\partial}{\partial x}+\boldsymbol{j} \frac{\partial}{\partial y}+\boldsymbol{k} \frac{\partial}{\partial z},
$$

is a differential operator with $\boldsymbol{i}, \boldsymbol{j}, \boldsymbol{k}$ denoting the unit vector in the $x, y, z$ direction, respectively.

Consider the nonlinear interaction of a steady-state wave system of two periodic travelling waves. Write

$$
\xi_{i}=\boldsymbol{k}_{i} \cdot \boldsymbol{r}-\sigma_{i} t, \quad i=1,2,
$$

where $\boldsymbol{k}_{i}$ is the wavenumber, $\sigma_{i}$ denotes actual wave frequency and $\boldsymbol{r}=x \boldsymbol{i}+y \boldsymbol{j}$, respectively. In this paper, we focus on the steady-state cases, say, all wavenumbers $\boldsymbol{k}_{i}$ and actual wave frequency $\sigma_{i}$, where $i=1,2$, are time-independent. Due to the nonlinearity, the actual wave frequency $\sigma_{i}$ is larger than the linear frequency $\omega_{i}=\sqrt{g\left|\boldsymbol{k}_{i}\right|}$. Thus, the wave elevation can be expressed in the form

$$
\eta\left(\xi_{1}, \xi_{2}\right)=\sum_{m=0}^{+\infty} \sum_{n=-\infty}^{+\infty} A_{m, n} \cos \left(m \xi_{1}+n \xi_{2}\right),
$$


where $A_{m, n}$ is a real number. Thus, $A_{m, n}^{2}$ versus $\left|m \boldsymbol{k}_{1}+n \boldsymbol{k}_{2}\right|$ gives a wave spectrum. Here,

$$
\frac{A_{m, n}^{2}}{\sum_{m=0}^{+\infty} \sum_{n=-\infty}^{+\infty} A_{m, n}^{2}}
$$

gives the percentage of wave energy of the wave component $\cos \left(m \xi_{1}+n \xi_{2}\right)$. As pointed out by Benney (1962), in a special case of exact resonance

$$
\boldsymbol{k}_{3}=2 \boldsymbol{k}_{1}-\boldsymbol{k}_{2}, \quad \omega_{3}=2 \omega_{1}-\omega_{2},
$$

there exist periodic exchanges of wave energy between different wave components, i.e. $A_{m, n}$ is time-dependent in general. In this case, the corresponding wave spectrum is time-dependent. Note that the wave spectrum is time-independent when $A_{m, n}, \boldsymbol{k}_{i}$ and $\sigma_{i}$ are constants, corresponding to the so-called 'steady-state waves'. In this paper, the wavenumber $\boldsymbol{k}_{i}$ and actual wave frequency $\sigma_{i}>\omega_{i}=\sqrt{g\left|\boldsymbol{k}_{i}\right|}$, where $i=1,2$, are given as constants, and the unknown, time-independent coefficient $A_{m, n}$ is determined in the way mentioned below. In addition, without loss of generality, we consider here only the so-called 'nearly resonant' case when (2.9) is nearly satisfied.

Since all of the wave amplitudes, the wavenumbers $\boldsymbol{k}_{i}$ and the actual wave frequencies $\sigma_{i}$ of the steady-state travelling waves are constant, i.e. independent of time, the original initial/boundary-value problem governed by (2.1)-(2.4) can be transformed into a boundary-value one by means of the new variables $\xi_{1}$ and $\xi_{2}$. In the new coordinate system $\left(\xi_{1}, \xi_{2}, z\right)$, the Laplace equation (2.1) reads

$$
\sum_{i=1}^{2} \sum_{j=1}^{2} \boldsymbol{k}_{i} \cdot \boldsymbol{k}_{j} \frac{\partial^{2} \varphi}{\partial \xi_{i} \partial \xi_{j}}+\frac{\partial^{2} \varphi}{\partial z^{2}}=0, \quad-\infty<z<\eta\left(\xi_{1}, \xi_{2}\right)
$$

subject to the two boundary conditions on the unknown wave elevation $z=\eta\left(\xi_{1}, \xi_{2}\right)$ :

$$
\begin{gathered}
\mathscr{N}_{1}[\varphi]=\sum_{i=1}^{2} \sum_{j=1}^{2} \sigma_{i} \sigma_{j} \frac{\partial^{2} \varphi}{\partial \xi_{i} \partial \xi_{j}}+g \frac{\partial \varphi}{\partial z}-2 \sum_{i=1}^{2} \sigma_{i} \frac{\partial f}{\partial \xi_{i}} \\
+\sum_{i=1}^{2} \sum_{j=1}^{2} \boldsymbol{k}_{i} \cdot \boldsymbol{k}_{j} \frac{\partial \varphi}{\partial \xi_{i}} \frac{\partial f}{\partial \xi_{j}}+\frac{\partial \varphi}{\partial z} \frac{\partial f}{\partial z}=0, \quad \text { on } z=\eta\left(\xi_{1}, \xi_{2}\right), \\
\mathscr{N}_{2}[\varphi, \eta]=\eta-\frac{1}{g}\left(\sum_{i=1}^{2} \sigma_{i} \frac{\partial \varphi}{\partial \xi_{i}}-f\right)=0, \quad \text { on } z=\eta\left(\xi_{1}, \xi_{2}\right),
\end{gathered}
$$

and also the impermeable condition at the bottom

$$
\lim _{z \rightarrow-\infty} \frac{\partial \varphi}{\partial z}=0
$$

where $\mathscr{N}_{1}$ and $\mathscr{N}_{2}$ are two nonlinear operators defined above, and

$$
f=\frac{1}{2}\left[\sum_{i=1}^{2} \sum_{j=1}^{2} \boldsymbol{k}_{i} \cdot \boldsymbol{k}_{j} \frac{\partial \varphi}{\partial \xi_{i}} \frac{\partial \varphi}{\partial \xi_{j}}+\left(\frac{\partial \varphi}{\partial z}\right)^{2}\right] .
$$


For a steady-state wave system with time-independent spectrum, there is no exchange of wave energy between different wave components, i.e. all physical quantities (such as wave amplitude and frequency) are constant. Thus, the steady-state wave elevation $\eta$ reads

$$
\eta\left(\xi_{1}, \xi_{2}\right)=\sum_{m=0}^{+\infty} \sum_{n=-\infty}^{+\infty} A_{m, n} \cos \left(m \xi_{1}+n \xi_{2}\right)
$$

where $A_{m, n}$ is a constant to be determined. Similarly, the velocity potential $\varphi\left(\xi_{1}, \xi_{2}, z\right)$ should be in the form

$$
\varphi\left(\xi_{1}, \xi_{2}, z\right)=\sum_{m=0}^{+\infty} \sum_{n=-\infty}^{+\infty} B_{m, n} \Psi_{m, n}\left(\xi_{1}, \xi_{2}, z\right)
$$

with the definition

$$
\Psi_{m, n}\left(\xi_{1}, \xi_{2}, z\right)=\sin \left(m \xi_{1}+n \xi_{2}\right) \exp \left(\left|m \boldsymbol{k}_{1}+n \boldsymbol{k}_{2}\right| z\right),
$$

where $B_{m, n}$ is a constant to be determined. Note that the linear governing equation (2.10) and the bottom condition (2.13) are automatically satisfied by (2.16). So, the unknown coefficients $A_{m, n}$ and $B_{m, n}$ are determined by the two nonlinear boundary conditions (2.11) and (2.12).

In the special case (2.9) of the exact resonance, Liao (2011) successfully applied the HAM to gain the (numerically) convergent analytic approximation solutions of the full wave equations for the steady-state exactly resonant waves in deep water with time-independent spectrum. The same approach was further used by $\mathrm{Xu}$ et al. (2012) to the full wave equations for the steady-state exactly resonant waves in finite water depth, and by Liu \& Liao (2014) to the full wave equation for steady-state exactly resonant waves in deep water in more general cases. The steady-state nearly resonant waves can be obtained in a rather similar way, as shown below. Since almost all mathematical formulae are the same as those given by Liao (2011), we only describe here this approach briefly. For details, please refer to appendix A.

In the HAM-based approach (Liao 2011), the velocity potential $\varphi\left(\xi_{1}, \xi_{2}, z\right)$ and the free surface elevation $\eta\left(\xi_{1}, \xi_{2}\right)$ are expressed by two infinite series

$$
\varphi\left(\xi_{1}, \xi_{2}, z\right)=\varphi_{0}\left(\xi_{1}, \xi_{2}, z\right)+\sum_{m=1}^{+\infty} \varphi_{m}\left(\xi_{1}, \xi_{2}, z\right), \quad \eta\left(\xi_{1}, \xi_{2}\right)=\sum_{m=1}^{+\infty} \eta_{m}\left(\xi_{1}, \xi_{2}\right)
$$

where

$$
\eta_{m}=c_{0} \Delta_{m-1}^{\eta}+\chi_{m} \eta_{m-1}, \quad \text { on } z=0,
$$

with the definition $\chi_{1}=0$ and $\chi_{m}=1$ for $m>1, \varphi_{0}\left(\xi_{1}, \xi_{2}, z\right)$ is an initial guess of the velocity potential, and $\varphi_{m}\left(\xi_{1}, \xi_{2}, z\right)(m \geqslant 1)$ is governed by the so-called $m$ th-order deformation equation

$$
\sum_{i=1}^{2} \sum_{j=1}^{2} \boldsymbol{k}_{i} \cdot \boldsymbol{k}_{j} \frac{\partial^{2} \varphi_{m}}{\partial \xi_{i} \partial \xi_{j}}+\frac{\partial^{2} \varphi_{m}}{\partial z^{2}}=0, \quad-\infty<z<0,
$$

subject to the linear boundary condition

$$
\mathscr{L}\left[\varphi_{m}\right]=c_{0} \Delta_{m-1}^{\phi}+\chi_{m} S_{m-1}-\bar{S}_{m}, \quad \text { on } z=0,
$$


and the bed condition

$$
\frac{\partial \varphi_{m}}{\partial z}=0, \quad \text { as } z \rightarrow-\infty .
$$

Here, $\mathscr{L}$ is an auxiliary linear operator and $c_{0}$ is an auxiliary parameter, called 'the convergence-control parameter'. In addition, all of the terms $\Delta_{m-1}^{\eta}, \Delta_{m-1}^{\phi}, S_{m-1}, \bar{S}_{m}$ on the right-hand side are determined by the known previous approximations $\eta_{j}$ and $\varphi_{j}$, where $j=0,1,2, \ldots, m-1$. For details, please refer to appendix A. Thus, as long as the initial guess $\varphi_{0}$ of the velocity potential, the auxiliary linear operator $\mathscr{L}$ and the convergence-control parameter $c_{0}$ are properly chosen, one can gain $\eta_{j}$ and $\varphi_{j}$ one by one.

It should be emphasized that, in the above-mentioned HAM-based approach (Liao 2011), we have great freedom to choose the auxiliary linear operator $\mathscr{L}$, the initial guesses $\varphi_{0}$ of the velocity potential and the convergence-control parameter $c_{0}$. As mentioned by Liao (2011), Xu et al. (2012) and Liu \& Liao (2014), it is the freedom of the HAM on the choice of the initial guess that provides us a way to handle the singularity of the steady-state exactly resonant waves. In addition, although the so-called 'convergence-control parameter' $c_{0}$ in (2.19) and (2.21) has no physical meanings, it provides us a simple way to guarantee the convergence of the solution series (2.18), as illustrated by Liao (2011), Xu et al. (2012) and Liu \& Liao (2014) for the steady-state exactly resonant waves.

For the steady-state exactly resonant water waves satisfying the criterion (2.9), Liao (2011) chose the following auxiliary linear operator

$$
\mathscr{L}[\varphi]=\omega_{1}^{2} \frac{\partial^{2} \varphi}{\partial \xi_{1}^{2}}+2 \omega_{1} \omega_{2} \frac{\partial^{2} \varphi}{\partial \xi_{1} \partial \xi_{2}}+\omega_{2}^{2} \frac{\partial^{2} \varphi}{\partial \xi_{2}^{2}}+g \frac{\partial \varphi}{\partial z}, \quad-\infty<z<\eta\left(\xi_{1}, \xi_{2}\right),
$$

which has the property

$$
\mathscr{L}\left[\Psi_{m, n}\left(\xi_{1}, \xi_{2}, z\right)\right]=\lambda_{m, n} \Psi_{m, n}\left(\xi_{1}, \xi_{2}, z\right), \quad-\infty<z<\eta\left(\xi_{1}, \xi_{2}\right)
$$

and

$$
\mathscr{L}^{-1}\left[\Psi_{m, n}\left(\xi_{1}, \xi_{2}, z\right)\right]=\frac{\Psi_{m, n}\left(\xi_{1}, \xi_{2}, z\right)}{\lambda_{m, n}}, \quad-\infty<z<\eta\left(\xi_{1}, \xi_{2}\right),
$$

where $\mathscr{L}^{-1}$ is the inverse operator of $\mathscr{L}, \Psi_{m, n}$ defined by (2.17) is the eigenfunction, and

$$
\lambda_{m, n}=g\left|m \boldsymbol{k}_{1}+n \boldsymbol{k}_{2}\right|-\left(m \omega_{1}+n \omega_{2}\right)^{2}
$$

is an eigenvalue of $\mathscr{L}$ for arbitrary wavenumber $\boldsymbol{k}_{i}$ and the linear frequency $\omega_{i}=\sqrt{g\left|\boldsymbol{k}_{i}\right|}$ of the primary waves, where $i=1,2$, with the property

$$
\lambda_{1,0}=\lambda_{0,1}=0
$$

In addition, in the case (2.9) for the exactly resonant waves, it holds

$$
\lambda_{2,-1}=0
$$

So, from a mathematical viewpoint, the exactly resonant wave system (2.9) has three eigenvalues to be zero, i.e.

$$
\lambda_{1,0}=\lambda_{0,1}=\lambda_{2,-1}=0 .
$$


Owing to (2.25), it is the additional zero eigenvalue (such as $\lambda_{2,-1}$ in the abovementioned case) that brings singularity to the exactly resonant wave problems. Such kind of singularity is mathematically difficult to handle in the frame of perturbation methods, as mentioned by Madsen \& Fuhrman (2012). However, it is easy to overcome in the frame of the HAM, as illustrated by Liao (2011), Xu et al. (2012) and Liu \& Liao (2014).

Write

$$
\delta_{m, n}=\left|\lambda_{m, n}\right|=|g| m \boldsymbol{k}_{1}+n \boldsymbol{k}_{2}\left|-\left(m \omega_{1}+n \omega_{2}\right)^{2}\right| .
$$

Thus,

$$
\lambda_{m, n}=0, \quad m^{2}+n^{2}>1
$$

corresponds to the exact resonance. So, it is reasonable to define

$$
0<\left|\lambda_{m, n}\right| \leqslant 10^{-3}, \quad m^{2}+n^{2}>1
$$

for the nearly resonant waves. Here $10^{-3}$ is a small number, which can be replaced by others such as $10^{-4}$, but the conclusions given in this paper are qualitatively the same.

From a mathematical viewpoint, when the resonance criterion (2.9) is nearly satisfied, we have two zero eigenvalues $\lambda_{1.0}$ and $\lambda_{0,1}$, and one eigenvalue $\lambda_{2,-1}$ very close to zero. In this case, the famous 'small divisor problem' (Poincaré 1892; Yoccoz 1992) arises, and the above-mentioned HAM-based approach for steady-state exactly resonant waves (Liao 2011; Xu et al. 2012; Liu \& Liao 2014) does not work, due to the same mathematical difficulties mentioned by Meiron et al. (1982), Craig \& Nicholls (2002) and Nicholls \& Reitich (2006) for three-dimensional periodic travelling waves.

How to overcome this 'small divisor problem' in the frame of the HAM?

Note that the HAM-based approach (Liao 2011; Xu et al. 2012; Liu \& Liao 2014) works well for the steady-state exactly resonant waves that have more than two zero eigenvalues. Can we transfer the nearly resonant wave problems into exactly resonant ones? If yes, the nearly resonant wave problems can be solved almost in the same way as the exactly resonant ones.

It should be emphasized that the HAM-based approach (Liao 2011; Xu et al. 2012; Liu \& Liao 2014) provides us great freedom to choose the auxiliary linear operator $\mathscr{L}$. For the steady-state exactly resonant waves in case of (2.9), Liao (2011) simply chose the auxiliary linear operator (2.23), which is exactly the linear part of (2.11). However, if we choose the same auxiliary linear operator (2.23) for the nearly resonant waves, the small divisor $\lambda_{m, n}$ appears due to (2.25). Fortunately, in the frame of the HAM, it is not absolutely necessary to do so: the freedom provided by the HAM is so large that, for the steady-state nearly resonant waves, we can choose here such a new, generalized auxiliary linear operator

$$
\mathscr{L}[\varphi]=\omega_{1}^{2} \frac{\partial^{2} \varphi}{\partial \xi_{1}^{2}}+\mu \omega_{1} \omega_{2} \frac{\partial^{2} \varphi}{\partial \xi_{1} \partial \xi_{2}}+\omega_{2}^{2} \frac{\partial^{2} \varphi}{\partial \xi_{2}^{2}}+g \frac{\partial \varphi}{\partial z}, \quad-\infty<z<\eta\left(\xi_{1}, \xi_{2}\right),
$$

where

$$
\mu=\frac{4 \omega_{1}^{2}+\omega_{2}^{2}-g\left|2 \boldsymbol{k}_{1}-\boldsymbol{k}_{2}\right|}{2 \omega_{1} \omega_{2}}
$$

is a constant in the case that (2.9) is nearly satisfied, with the property

$$
\mathscr{L}\left[\Psi_{m, n}\left(\xi_{1}, \xi_{2}, z\right)\right]=\bar{\lambda}_{m, n} \Psi_{m, n}\left(\xi_{1}, \xi_{2}, z\right), \quad-\infty<z<\eta\left(\xi_{1}, \xi_{2}\right),
$$


and

$$
\mathscr{L}^{-1}\left[\Psi_{m, n}\left(\xi_{1}, \xi_{2}, 0\right)\right]=\frac{\Psi_{m, n}\left(\xi_{1}, \xi_{2}, z\right)}{\bar{\lambda}_{m, n}}, \quad-\infty<z<\eta\left(\xi_{1}, \xi_{2}\right),
$$

in which $\Psi_{m, n}$ defined by (2.17) is the eigenfunction,

$$
\bar{\lambda}_{m, n}=g\left|m \boldsymbol{k}_{1}+n \boldsymbol{k}_{2}\right|-\left(m^{2} \omega_{1}^{2}+\mu m n \omega_{1} \omega_{2}+n^{2} \omega_{2}^{2}\right)
$$

is an eigenvalue of the generalized auxiliary linear operator (2.33), respectively.

This is mainly because we have great freedom to choose the auxiliary linear operator in the frame of the HAM-based approach (Liao 2011). Note that, for the nearly resonant waves with the small eigenvalue $0<\left|\lambda_{2,-1}\right| \leqslant 10^{-3}$, there are only two zero eigenvalues $\lambda_{1,0}=\lambda_{0,1}=0$, where $\lambda_{m, n}$ defined by (2.26) is the eigenvalue of the auxiliary linear operator (2.23) that comes from the linear part of the original wave equations. However, using the generalized auxiliary linear operator (2.33), we have now three zero eigenvalues

$$
\bar{\lambda}_{1,0}=\bar{\lambda}_{0,1}=\bar{\lambda}_{2,-1}=0
$$

where $\bar{\lambda}_{m, n}$ is the eigenvalue of (2.33), with the same eigenfunction $\Psi_{m, n}$ defined by (2.17). In this way, the small divisor (due to small eigenvalue such as $\lambda_{2,-1}$ ) at low frequency is avoided, or at least moved far away to rather high frequency with physically negligible wave energy, as illustrated below.

In the case (2.9) of the exactly resonant waves with $\lambda_{2,-1}=0$, corresponding to

$$
4 \omega_{1} \omega_{2}=4 \omega_{1}^{2}+\omega_{2}^{2}-g\left|2 \boldsymbol{k}_{1}-\boldsymbol{k}_{2}\right|
$$

due to the definition (2.26), we have $\mu=2$ according to the definition (2.34). However, when (2.9) is nearly satisfied, $\mu$ deviates from 2 a little, so that the generalized auxiliary linear operator (2.33) is a little different from (2.23) used by Liao (2011) for the exactly resonant waves. Therefore, the auxiliary linear operator (2.33) works not only for the nearly resonant cases (such as $0<\left|\lambda_{2,-1}\right| \leqslant 10^{-3}$ ) but also for exactly resonant ones (such as $\lambda_{2,-1}=0$ ), since all other mathematical formulae are the same (Liao 2011), as described in appendix A. In other words, the auxiliary linear operator (2.23) used by Liao (2011) for the exactly resonant waves is only a special case of the new auxiliary linear operator (2.33) for the nearly resonant ones. Thus, as long as the generalized auxiliary linear operator (2.33) is used, there always exist three zero eigenvalues

$$
\bar{\lambda}_{1,0}=\bar{\lambda}_{0,1}=\bar{\lambda}_{2,-1}=0
$$

no matter whether the criterion (2.9) is either exactly or nearly satisfied. In other words, we can solve the steady-state nearly resonant waves almost in the same way as the exactly resonant ones. Therefore, from the viewpoints of the HAM, the steady-state nearly resonant water waves have nothing fundamentally different from the exactly resonant waves, except the more general definition (2.33) of the auxiliary linear operator $\mathscr{L}$. We will illustrate this point in $\S 3$ by means of a case study.

In summary, our strategy is to transfer the nearly resonant wave problem (with two zero eigenvalues $\bar{\lambda}_{1,0}=\bar{\lambda}_{0,1}=0$ and one small eigenvalue $\bar{\lambda}_{2,-1}$ that leads to small divisor problem) into the corresponding exactly resonant wave problem with three zero eigenvalues

$$
\bar{\lambda}_{1,0}=\bar{\lambda}_{0,1}=\bar{\lambda}_{2,-1}=0
$$


by means of choosing a generalized auxiliary linear operator (2.33) in the frame of the HAM. Note that the steady-state exactly resonant travelling waves in deep water and in finite depth governed by the full wave equations were successfully solved by means of the HAM (Liao 2011; Xu et al. 2012; Liu \& Liao 2014), and also observed experimentally in a laboratory with good agreement between analytic approximation and experimental results (Liu et al. 2015). Here, it should be emphasized that, it is the HAM that provides us such kind of freedom to choose the generalized auxiliary linear operator (2.33), which is different from the linear part (2.23) of the original wave equations. This is quite different from perturbation methods (Madsen \& Fuhrman 2012) and the numerical approaches (Meiron et al. 1982; Craig \& Nicholls 2002; Nicholls \& Reitich 2006), which always regard the linear part (2.23) of the original wave equations as a linear operator. As a result, perturbation methods and these numerical approaches had to directly handle the small divisor problem, which is mathematically very difficult to overcome, as pointed out by Meiron et al. (1982), Craig \& Nicholls (2002), Nicholls \& Reitich (2006) and Madsen \& Fuhrman (2012). This is the novelty value of our HAM-based approach for the steady-state nearly resonant travelling waves.

\section{A case study}

Without loss of generality, we consider here such a case that the resonance criterion (2.9) is satisfied either exactly or nearly, which can be solved by means of the HAM-based approach (Liao 2011) using the generalized auxiliary linear operator (2.33). Moreover, without loss of generality, we consider a special case

$$
\alpha=\frac{\pi}{36}, \quad \frac{\sigma_{1}}{\omega_{1}}=\frac{\sigma_{2}}{\omega_{2}}=\epsilon, \quad k_{2}=\frac{\pi}{5}, \quad 0.89 \leqslant \frac{k_{2}}{k_{1}} \leqslant 0.895,
$$

where $\alpha$ denotes the angle between the wavenumbers $\boldsymbol{k}_{1}$ and $\boldsymbol{k}_{2}, \sigma_{i}$ is the actual frequency, $\omega_{i}=\sqrt{g k_{i}}$ with $k_{i}=\left|\boldsymbol{k}_{i}\right|$ is the linear frequency and $\epsilon$ is a constant, respectively. Note that the resonance criterion (2.9) is exactly satisfied when $k_{2} / k_{1}=0.8925$, a case studied by Liao (2011) in detail. Except the generalized auxiliary linear operator (2.33), all other formulae are exactly the same as those for the steady-state exactly resonant waves (Liao 2011), as mentioned in appendix A. The (numerically) convergent analytic approximations of the steady-state wave systems for $0.89 \leqslant k_{2} / k_{1} \leqslant 0.895$ in the case of (3.1) with $\epsilon=1.0003$ are obtained almost in the same way to that described by Liao (2011) for the steady-state exactly resonant waves. For details, please refer to appendix A.

We emphasize here that, unlike perturbation techniques and other analytic approximation methods, our HAM-based approach contains the so-called 'convergencecontrol parameter' $c_{0}$, which has no physical meanings but can provide us a convenient way to guarantee the convergence of solution series. For example, in case of $k_{2} / k_{1}=0.8915$ (corresponding to a case of nearly resonant waves), the solution series converges (strictly speaking, here it is a kind of 'numerical' convergence, since we just numerically show the decrease of residual errors versus the order of approximation, which however is not a mathematical proof), rather quickly by means of $-1.25 \leqslant c_{0} \leqslant-0.75$, with the optimal value of $c_{0}$ near -0.95 , as shown in table 1 . In fact, the numerical convergence of solution series given by the HAM for exactly resonant waves was generally guaranteed in the same way, as shown by Liao (2011), Xu et al. (2012), Liu \& Liao (2014) and Liu et al. (2015). Note that the numerically convergent analytic approximations for the steady-state exactly resonant waves in 
Order of approximation 1

3

5

10

15

20

\begin{tabular}{cc}
\multicolumn{2}{c}{$c_{0}=-1.25$} \\
\hline $\mathscr{E}_{\phi}$ & $\mathscr{E}_{\eta}$ \\
$9.5 \times 10^{-8}$ & $7.8 \times 10^{-4}$ \\
$3.8 \times 10^{-9}$ & $3.2 \times 10^{-6}$ \\
$2.3 \times 10^{-10}$ & $7.0 \times 10^{-8}$ \\
$8.2 \times 10^{-13}$ & $4.2 \times 10^{-10}$ \\
$3.7 \times 10^{-15}$ & $3.1 \times 10^{-13}$ \\
$3.5 \times 10^{-18}$ & $1.0 \times 10^{-14}$
\end{tabular}

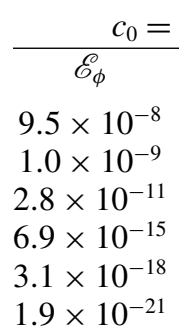

$c_{0}=-0.95$

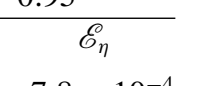

$7.8 \times 10^{-4}$

$1.5 \times 10^{-7}$

$3.4 \times 10^{-9}$

$4.3 \times 10^{-13}$

$1.4 \times 10^{-16}$

$8.6 \times 10^{-20}$

\begin{tabular}{cc}
\multicolumn{2}{c}{$c_{0}=-0.75$} \\
\hline $\mathscr{E}_{\phi}$ & $\mathscr{E}_{\eta}$ \\
$9.5 \times 10^{-8}$ & $7.8 \times 10^{-4}$ \\
$2.8 \times 10^{-9}$ & $2.9 \times 10^{-6}$ \\
$2.3 \times 10^{-10}$ & $2.7 \times 10^{-8}$ \\
$5.2 \times 10^{-13}$ & $4.7 \times 10^{-11}$ \\
$1.5 \times 10^{-15}$ & $9.7 \times 10^{-14}$ \\
$5.6 \times 10^{-18}$ & $2.7 \times 10^{-16}$
\end{tabular}

TABLE 1. Averaged residual errors versus order of approximation for nearly resonant waves in case of $\sigma_{1} / \sqrt{g k_{1}}=\sigma_{2} / \sqrt{g k_{2}}=1.0003$ and $k_{2} / k_{1}=0.8915$ by means of different values of the convergence-control parameter $c_{0}$, with the corresponding distribution of wave energy $81.9 \%$ (the first primary wave component), $9.7 \%$ (the second primary one) and $8.2 \%$ (the nearly resonant one). The angle between two wavenumbers $\boldsymbol{k}_{1}$ and $\boldsymbol{k}_{2}$ of the two primary waves is $\pi / 36$. $\mathscr{E}_{\phi}$ and $\mathscr{E}_{\eta}$ denote the averaged residual errors of the two boundary conditions on the free surface.

deep water given by the HAM-based approach agreed well with the experimental observation in a laboratory, as mentioned by Liu et al. (2015).

In addition, unlike perturbation techniques, the HAM provides us great freedom to choose auxiliary linear operator $\mathscr{L}$. The original linear operator (2.23) is widely used in perturbation approaches, which unfortunately leads to the so-called 'small divisor problem' at a low frequency (say, $m=2, n=-1$, corresponding to the wave component $\left.\cos \left(2 \xi_{1}-\xi_{2}\right)\right)$ for the nearly resonant case considered in this paper. Such kind of 'small divisor problem' is very difficult to overcome in mathematics, as pointed out by Meiron et al. (1982), Craig \& Nicholls (2002), Nicholls \& Reitich (2006) and Madsen \& Fuhrman (2012). However, by means of simply choosing a new auxiliary linear operator (2.33) in the frame of the HAM, such kind of 'small divisor problem' can be easily avoided: it is found that, in the case considered in table 1, the so-called 'small divisor problem', corresponding to $\delta_{m, n}<10^{-3}$ with the definition (2.30), does not occur at least for

$$
0<m \leqslant 10^{5}, \quad|n| \leqslant 10^{5}
$$

by means of using the generalized auxiliary linear operator (2.33). From physical viewpoints, the kinetic energy of wave components with very high frequency, such as

$$
A_{m, n} \cos \left(m \xi_{1}+n \xi_{2}\right), \quad|m|>10^{5} \text { and/or }|n|>10^{5},
$$

is rather small and thus can be ignored. Therefore, in the frame of the HAM, the small divisor problem does not appear even at the 1000th-order of approximations. Note that such high-order analytic approximations are unnecessary from numerical viewpoint, since the averaged residual errors of the two boundary conditions are at the level of $10^{-20}$ by means of the optimal convergence-control parameter $c_{0}=-0.95$, as shown in table 1. In this way, the 'small divisor' at the low frequency of the steady-state nearly resonant waves is successfully moved far away to much higher frequencies, or even to infinity (although we cannot strictly prove this point right now for the considered case). This is mainly because the HAM is fundamentally different from other perturbation approaches, since it has nothing to do with any small physical parameters at all. Note that all other perturbation approaches cannot avoid such kind 
$k_{2} / k_{1} \quad$ First primary wave Second primary wave Exactly or nearly resonant wave

(\%)

$0.89 \quad 6.3$

$0.89 \quad 54.0$

0.8905

0.8905

0.891

0.891

0.891

0.891

0.8915

0.8915

0.8915

0.8915

0.892

0.892

0.892

0.892

$0.8925^{*}$

$0.8925^{*}$

$0.8925^{*}$

0.893

0.893

0.893

0.8935

0.8935

0.8935

0.894

0.894

0.8945

0.8945

0.895
17.2

52.1

25.6

69.2

49.8

32.0

81.9

31.8

47.1

22.3

91.2

36.5

43.8

15.7

40.2

39.9

10.5

43.1

35.3

6.3

45.4

30.0

2.8

47.4

23.8

49.0

16.6

50.2
(\%)

67.4

28.2

62.0

26.7

58.0

14.8

25.0

18.2

9.7

55.1

23.0

16.3

5.1

52.9

20.8

14.2

51.1

18.3

12.1

49.6

15.6

10.0

48.3

12.5

7.9

46.9

9.1

45.1

5.5

42.4
(\%)

26.0

17.0

20.2

20.3

15.7

15.8

24.1

49.6

8.2

12.3

28.6

61.3

3.7

9.6

33.9

69.9

7.5

40.1

77.1

5.9

47.1

83.4

4.5

55.3

89.0

3.3

64.7

2.1

75.6

1.1

TABLE 2. Energy distribution of the steady-state exactly or nearly resonant waves in the case of $\sigma_{1} / \sqrt{g k_{1}}=\sigma_{2} / \sqrt{g k_{2}}=\epsilon=1.0003$ with various $k_{2} / k_{1}$. The angle between two wavenumbers $\boldsymbol{k}_{1}$ and $\boldsymbol{k}_{2}$ of the two primary waves is $\pi / 36$, and * denotes the exactly resonant wave.

of 'small divisor problems'. In other words, our HAM-based approach for the nearly resonant waves is novel, even only from the viewpoint that it can easily move the 'small divisors' at low frequency far away to rather high frequency by means of simply 


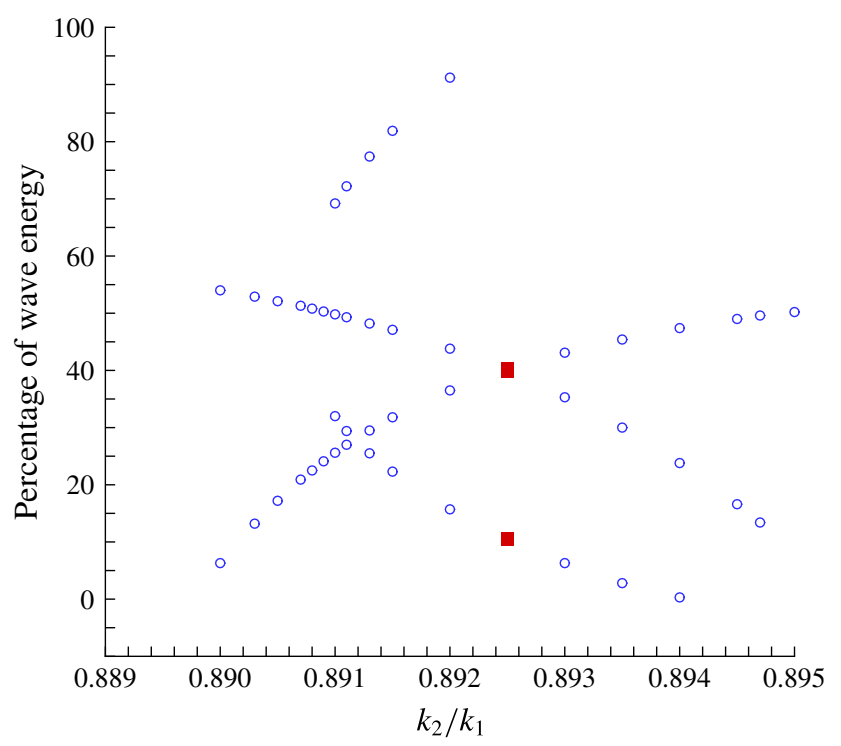

FIGURE 1. (Colour online) Wave energy distribution (\%) of the first primary wave of the steady-state exactly and nearly resonant wave system in the case of $\sigma_{1} / \sqrt{g k_{1}}=\sigma_{2} / \sqrt{g k_{2}}=$ $\epsilon=1.0003$ with various $k_{2} / k_{1}$, when the angle between wavenumbers $\boldsymbol{k}_{1}$ and $\boldsymbol{k}_{2}$ of the two primary waves is $\pi / 36$. The filled squares correspond to the exactly resonant wave.

choosing the generalized auxiliary linear operator (2.33) that is different from the linear part (2.23) of the original wave equations.

Here, we use another extraordinary advantage of the HAM, i.e. its freedom on choice of equation type for high-order approximations. In fact, such kind of advantage of the HAM has been successfully applied to many nonlinear problems, as illustrated by Liao \& Tan (2007) who applied the HAM to successfully solve a second-order two-dimensional Gelfand equation even by means of a fourth-order auxiliary linear operator, and a second-order three-dimensional Gelfand equation even by means of a sixth-order auxiliary linear operator, respectively. In this paper, we further illustrate that, by means of simply choosing the generalized auxiliary linear operator (2.33) in the frame of the HAM, the so-called 'small divisor' can be easily moved far away from the low frequency to a very high one, where kinetic energy of wave components can be ignored from a physical viewpoint.

Given a value of $k_{2} / k_{1}$, we search for the corresponding steady-state nearly (or exactly) resonant waves by means of the HAM-based approach mentioned above, and then gain the distribution of wave energy by means of (2.8). It is found that there exist multiple solutions in most cases, as shown in figures $1-3$. The energy distribution (\%) of the two primary waves (with the wavenumbers $\boldsymbol{k}_{1}$ and $\boldsymbol{k}_{2}$ ) and the nearly (or exactly) resonant wave component (with the wavenumber $\boldsymbol{k}_{3}=2 \boldsymbol{k}_{1}-\boldsymbol{k}_{2}$ ) is given in table 2.

Note that the resonance criterion (2.9) is exactly satisfied when $k_{2} / k_{1}=0.8925$ in the case of (3.1). The results of the exact resonance are marked by the filled squares in figures 1-3. It is very interesting that the exactly resonant results are not fundamentally different from other nearly resonant ones: from figures 1-3, one cannot distinguish the exactly resonant results from the nearly resonant ones. It suggests that, from physical viewpoints, nothing is fundamentally different between the steady-state 


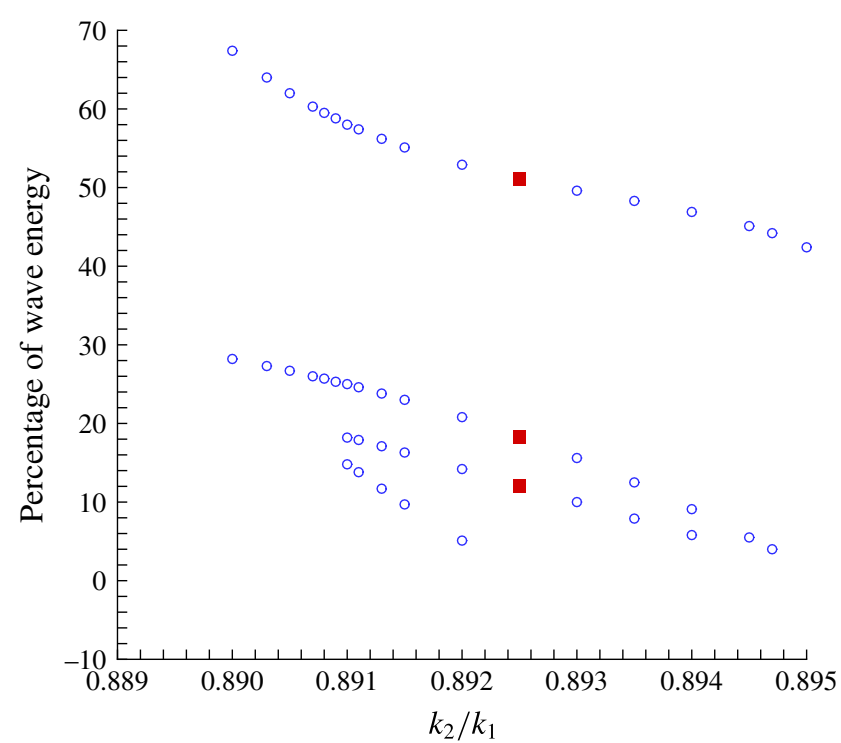

FIgURE 2. (Colour online) Wave energy distribution (\%) of the second primary wave of the steady-state exactly and nearly resonant wave system in the case of $\sigma_{1} / \sqrt{g k_{1}}=$ $\sigma_{2} / \sqrt{g k_{2}}=\epsilon=1.0003$ with various $k_{2} / k_{1}$, when the angle between wavenumbers $\boldsymbol{k}_{1}$ and $\boldsymbol{k}_{2}$ of the two primary waves is $\pi / 36$. The filled squares correspond to the exactly resonant wave.

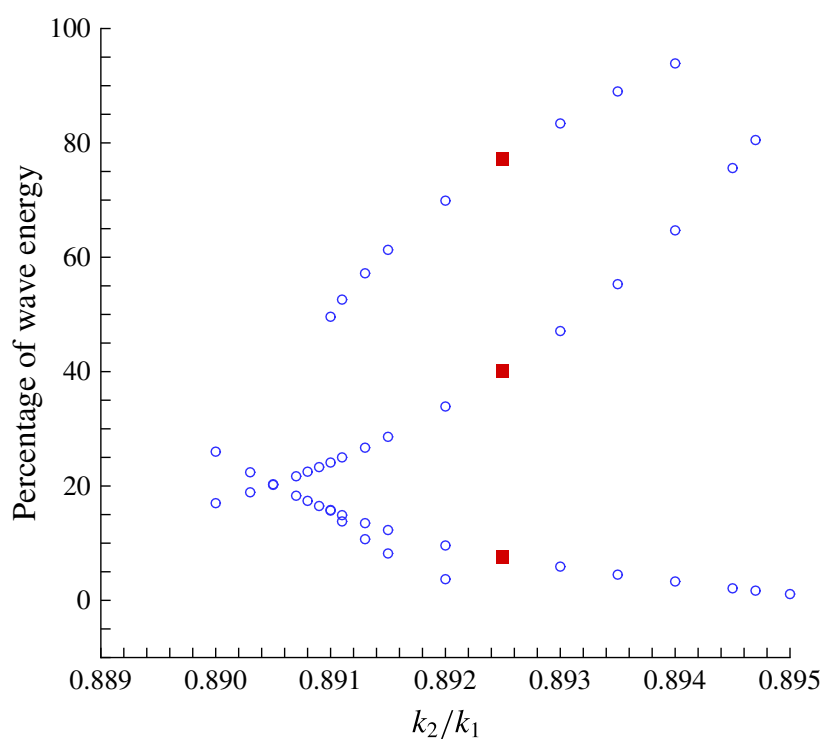

FIgURE 3. (Colour online) Wave energy distribution (\%) of the wave component $\boldsymbol{k}_{3}=$ $2 \boldsymbol{k}_{1}-\boldsymbol{k}_{2}$ of the steady-state exactly and nearly resonant wave system in the case of $\sigma_{1} / \sqrt{g k_{1}}=\sigma_{2} / \sqrt{g k_{2}}=\epsilon=1.0003$ with various $k_{2} / k_{1}$, when the angle between wavenumbers $\boldsymbol{k}_{1}$ and $\boldsymbol{k}_{2}$ of the two primary waves is $\pi / 36$. The filled squares correspond to the exactly resonant wave. 


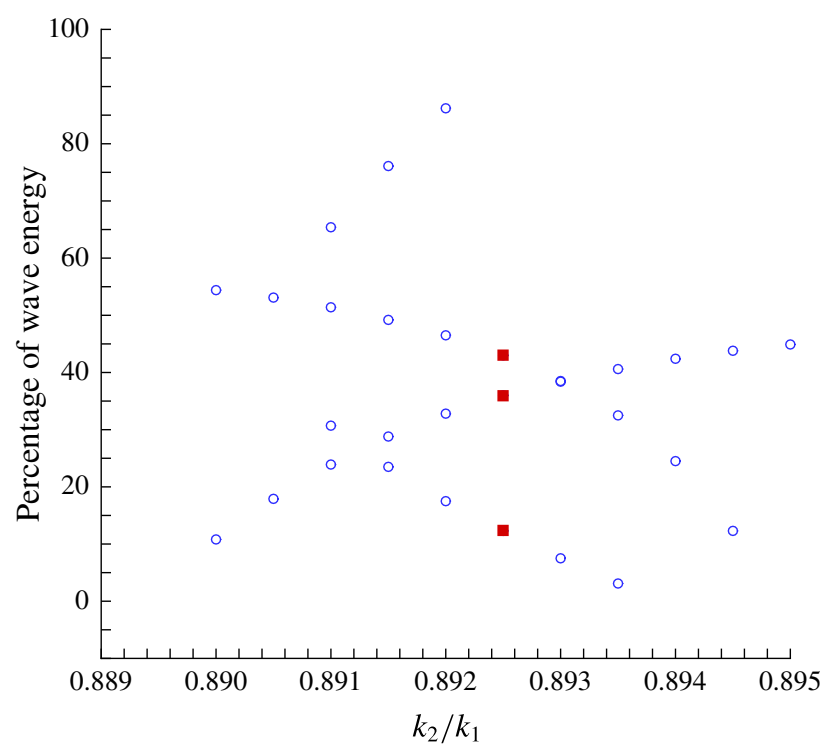

FIGURE 4. (Colour online) Wave energy distribution (\%) obtained by Zakharov equation of the first primary wave of the steady-state exactly and nearly resonant wave system in the case of $\sigma_{1} / \sqrt{g k_{1}}=\sigma_{2} / \sqrt{g k_{2}}=\epsilon=1.0003$ with various $k_{2} / k_{1}$, when the angle between wavenumbers $\boldsymbol{k}_{1}$ and $\boldsymbol{k}_{2}$ of the two primary waves is $\pi / 36$. The filled squares correspond to the exactly resonant wave.

exactly resonant waves and the steady-state nearly resonant ones. Note also that, mathematically speaking, both of the exactly and nearly resonant results are obtained in the same way by means of the same auxiliary linear operator (2.33) in the frame of the HAM. Therefore, from numerical viewpoints, nothing is fundamentally different between the exactly resonant waves and the nearly resonant ones either.

We studied many cases different from (3.1) in a similar way, and always obtained the corresponding steady-state nearly resonant waves. In all of our considered cases, the results of steady-state exactly resonant waves have nothing fundamentally different from those of nearly resonant ones. Thus, from physical viewpoints and in the frame of the HAM, nothing is fundamentally different between the steady-state exactly and nearly resonant waves.

All of our above-mentioned conclusions are based on the HAM-based approach for the full wave equations in deep water. Like Xu et al. (2012), we further numerically solve the Zakharov's equation so as to verify the correctness of our above-mentioned conclusions. The numerical approach based on the Zakharov's equation is described briefly in appendix B. For the same cases, numerical results given by the Zakharov's equation are fundamentally the same as the analytical ones given by our HAM-based approach for the full wave equations, as shown in figures 4-6. In particular, from a physical viewpoint, the results of the steady-state exactly resonant waves (marked by the filled squares) have nothing fundamentally different from those of the nearly resonant ones either. This verifies the validity of the above-mentioned HAM-based approach and our analytic approximations gained from the full wave equations. 


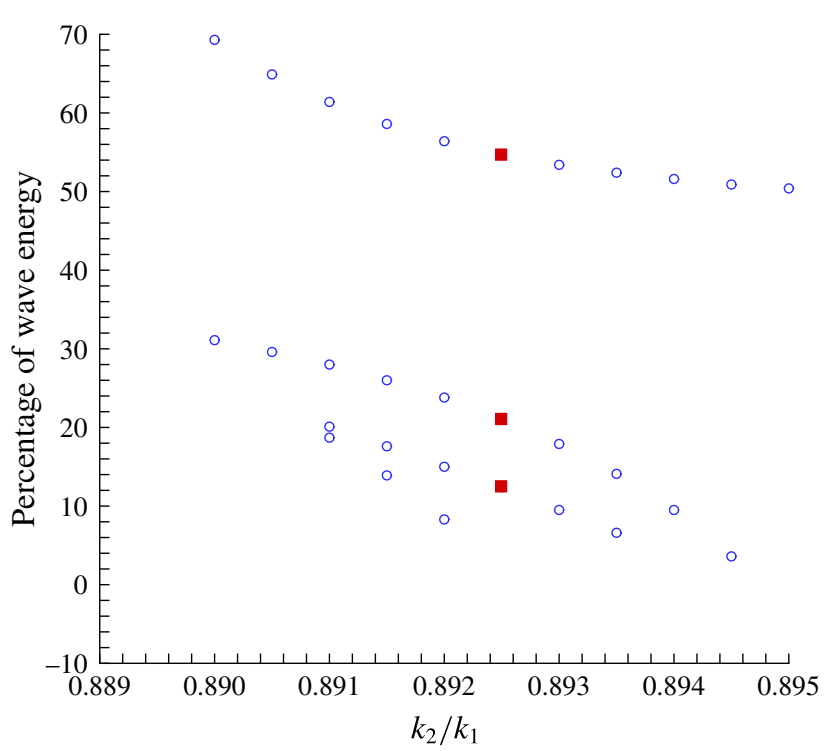

FIGURE 5. (Colour online) Wave energy distribution (\%) obtained by Zakharov equation of the second primary wave of the steady-state exactly and nearly resonant wave system in case of $\sigma_{1} / \sqrt{g k_{1}}=\sigma_{2} / \sqrt{g k_{2}}=\epsilon=1.0003$ with various $k_{2} / k_{1}$, when the angle between wavenumbers $\boldsymbol{k}_{1}$ and $\boldsymbol{k}_{2}$ of the two primary waves is $\pi / 36$. The filled squares correspond to the exactly resonant wave.

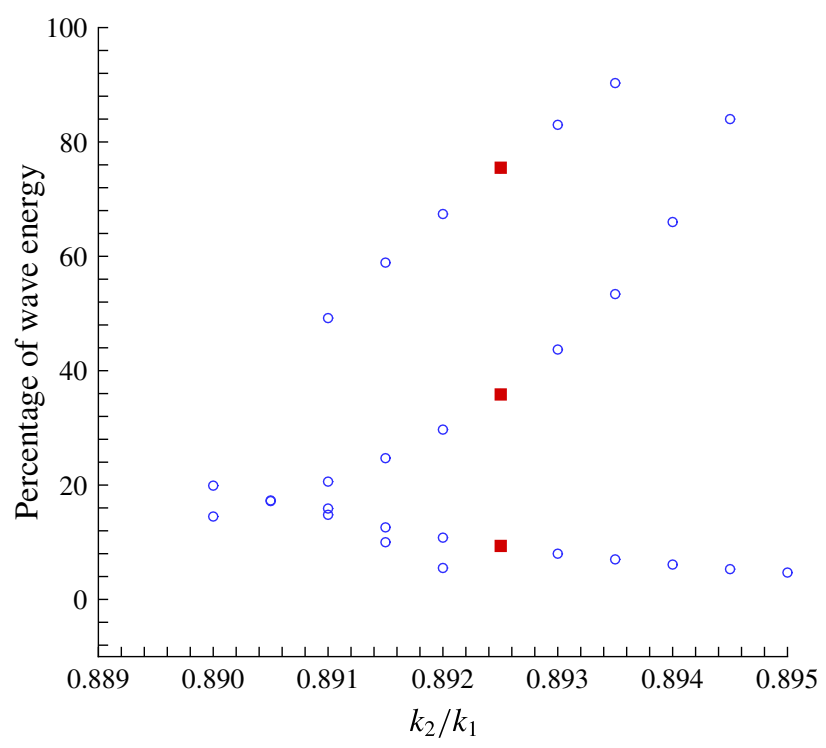

FIGURE 6. (Colour online) Wave energy distribution (\%) obtained by Zakharov equation of the wave component $\boldsymbol{k}_{3}=2 \boldsymbol{k}_{1}-\boldsymbol{k}_{2}$ of the steady-state exactly and nearly resonant wave system in the case of $\sigma_{1} / \sqrt{g k_{1}}=\sigma_{2} / \sqrt{g k_{2}}=\epsilon=1.0003$ with various $k_{2} / k_{1}$, when the angle between wavenumbers $\boldsymbol{k}_{1}$ and $\boldsymbol{k}_{2}$ of the two primary waves is $\pi / 36$. The filled squares correspond to the exactly resonant wave. 


\section{Concluding remarks and discussions}

In this paper, the HAM-based approach given by Liao (2011) for the steady-state exactly resonant waves is generalized for the steady-state nearly resonant waves by simply choosing the generalized auxiliary linear operator (2.33) but keeping all other formulae the same. In this way, we successfully gained, for the first time, the steadystate nearly resonant waves with time-independent spectrum in deep water. To the best of the authors' knowledge, this kind of steady-state nearly resonant wave has not been obtained analytically from the full wave equations. Therefore, considering the published results about the steady-state exactly resonant waves (Liao 2011; Xu et al. 2012, 2015; Liu \& Liao 2014; Liu et al. 2015), steady-state gravity waves with time-independent amplitudes widely exist, no matter whether the resonance criterion is exactly or nearly satisfied.

Note that the HAM-based approach for the exactly resonant waves does not work for the nearly resonant ones, since the famous 'small divisor problem' appears. Even for numerical approaches, the small divisor problem is rather difficult to overcome in mathematics, as pointed out by Meiron et al. (1982), Craig \& Nicholls (2002) and Nicholls \& Reitich (2006). Our strategy is to mathematically transfer the steadystate nearly resonant wave problem into the corresponding exactly resonant ones by means of choosing a more general auxiliary linear operator (2.33) than the linear part (2.23) of the original wave equations. In this way, the 'small divisor problem' at low frequency is moved far away to rather high frequency with physically negligible wave energy, such as the wave component

$$
A_{m, n} \cos \left(m \xi_{1}+n \xi_{2}\right), \quad|m|>10^{5} \text { and/or }|n|>10^{5}
$$

with a tiny wave amplitude $A_{m, n}$ much smaller than the small divisor, illustrated in a case study in $\S 3$. Note that it is the HAM that provides us such kind of freedom to choose the generalized auxiliary linear operator (2.33): perturbation and numerical approaches often directly use the linear part (2.23) of the original wave equations, and thus had to directly handle the small divisor problems. So, our HAM-based analytic approximation approach is fundamentally different from other perturbation methods (Madsen \& Fuhrman 2012) and numerical approaches (Meiron et al. 1982; Craig \& Nicholls 2002; Nicholls \& Reitich 2006). This also shows the novelty and originality of our approach for the steady-state nearly resonant travelling waves.

When the resonance criterion (2.9) is exactly satisfied, the general auxiliary linear operator (2.33) is the same (with $\mu=2$ ) as (2.23) for the exact resonance (Liao 2011), with three eigenvalues being zero. However, using the more general auxiliary linear operator (2.33), we always have three eigenvalues being zero, even in the case of the nearly resonant waves. So, from the viewpoint of the HAM, nothing is fundamentally different between the steady-state exactly and nearly resonant waves. Moreover, from figures 1-3, one cannot distinguish the results of the exact resonance from all other near-resonances. So, from the physical viewpoint, the results of the steady-state exactly resonant waves also have nothing fundamentally different from the nearly resonant ones, either.

Therefore, from the physical viewpoints and in the frame of the HAM, nothing is fundamentally different between the steady-state exactly/nearly resonant waves with time-independent spectrum. Then, a question arises: why should we define the socalled 'exactly resonant waves'? Let us consider such a thought experiment. Suppose that a person, who does not know Phillips' criterion of wave resonance at all, applies the above-mentioned HAM-based approach to solve the steady-state travelling waves 
with time-independent spectrum. For them, the results in the case (2.9) at $k_{2} / k_{1}=$ 0.8925 have nothing special at all from physical viewpoints. In addition, in the frame of the HAM, all results are gained by using the same mathematical formulae with the same auxiliary linear operator (2.33). Thus, it seems unnecessary for them to define the special case $k_{2} / k_{1}=0.8925$, which is traditionally called the 'exactly resonant waves' in the frame of perturbation methods. Note that the physics behind the same experimental observations should be independent of the used mathematical methods. So, this thought experiment suggests that the essence of the so-called 'wave resonance' should be reconsidered carefully from both physical and mathematical viewpoints.

The KP equation is a simplified model for water waves in shallow water. As pointed out by Hammack et al. $(1989,2005)$ and Dubrovin et al. (1997), the KP equation has a closed-form solution with arbitrary two phase variables. Thus, there exist the steadystate exactly and nearly resonant waves of the KP equation in shallow water, although Hammack et al. $(1989,2005)$ and Dubrovin et al. (1997) did not mention the wave resonance at all. However, the KP equation is only valid for waves in shallow water, and thus cannot describe waves in deep water. For example, Dubrovin et al. (1997) gave closed-form analytic solutions of the KP equation with three phases for travelling waves in shallow water, and found that 'almost every three-phase solution is timedependent, in every coordinate system'. This conclusion is not correct for waves in deep water, since rather complicated steady-state exactly resonant waves in deep water are indeed found by Liu \& Liao (2014) for the full wave equations. To the best of the authors' knowledge, the steady-state nearly resonant waves in deep water with timeindependent spectrum have never been obtained from the full wave equations in the form of analytic approximations. Neither have they been gained by other perturbation techniques (Madsen \& Fuhrman 2012) and numerical approaches due to the small divisor problem, as mentioned by Meiron et al. (1982), Craig \& Nicholls (2002) and Nicholls \& Reitich (2006).

Certainly, a lot of work should be done in future. Similarly, it is straightforward to gain the steady-state nearly resonant waves in a constant water depth and over a bottom with an infinite number of periodic ripples, in a similar manner to $\mathrm{Xu}$ et al. (2012) and $\mathrm{Xu}$ et al. (2015) for the exactly resonant ones. In addition, it is quite possible to observe the steady-state nearly resonant waves in deep water experimentally (For this purpose, the steady-state nearly resonant water waves with large enough nonlinearity should be used.) in a basin, just like Liu et al. (2015) did for the steady-state exactly resonant waves.

The HAM-based analytic approximation approach can successfully avoid the small-divisor problem (related to the nearly resonant wave propagation) by means of moving small divisor at low frequency far away to rather high frequencies with physically negligible wave energy. This is reasonable from physical and numerical viewpoints. However, from strictly mathematical viewpoints, the small divisor might be still an obstacle, since it is even unknown whether or not a solution exists mathematically (Craig \& Nicholls (2000) gave a rigorous proof of an existence theorem for two- and three-dimensional travelling water waves in the presence of capillarity. No existence theorems have been strictly proved for water waves in general, to the best of the authors' knowledge). This is similar to the famous Navier-Stokes equations, whose numerical solutions are widely used, but solution existence of the Navier-Stokes equations is not strictly proved mathematically in general.

\section{Acknowledgements}

Sincere thanks are due to the anonymous reviewers for their helpful comments. This work is partly supported by the National Natural Science Foundation of China 
(approval numbers 11272209 and 11432009) and National Key Basic Research Program of China (approval number 2014CB046801). This research was also supported by the Israel Science Foundation (grant number 464/13).

\section{Appendix A. Detailed mathematical formulae}

The full water wave equations (2.10)-(2.13) for the steady-state nearly resonant waves in deep water can be solved by means of the HAM nearly in the same way as that mentioned by Liao (2011). Shortly speaking, our strategy is to transfer the steady-state nearly resonant waves into a steady-state exactly resonant ones, by means of choosing a generalized auxiliary linear operator that is a little different from the linear part of the original wave equations.

Let $\varphi_{0}\left(\xi_{1}, \xi_{2}, z\right)$ denote a initial guess solution of velocity potential $\varphi$ satisfying the Laplace equation (2.10) and the bottom boundary condition (2.13), $\mathscr{L}$ an auxiliary linear operator with the property $\mathscr{L}[0]=0$, and $q \in[0,1]$ an embedding parameter without physical meaning, respectively. In the frame of the HAM, we first construct a family of solution $\Phi\left(\xi_{1}, \xi_{2}, z ; q\right)$ and $\zeta\left(\xi_{1}, \xi_{2} ; q\right)$ in $q \in[0,1]$ by means of the so-called zeroth-order deformation equation

$$
\sum_{i=1}^{2} \sum_{j=1}^{2} \boldsymbol{k}_{i} \cdot \boldsymbol{k}_{j} \frac{\partial^{2} \Phi}{\partial \xi_{i} \partial \xi_{j}}+\frac{\partial^{2} \Phi}{\partial z^{2}}=0, \quad-\infty<z<\zeta\left(\xi_{1}, \xi_{2} ; q\right)
$$

subject to the two boundary conditions on the unknown wave elevation $z=$ $\zeta\left(\xi_{1}, \xi_{2} ; q\right)$ :

$$
\begin{gathered}
(1-q) \mathscr{L}\left[\Phi\left(\xi_{1}, \xi_{2}, z ; q\right)-\varphi_{0}\left(\xi_{1}, \xi_{2}, z\right)\right]=c_{0} q \mathscr{N}_{1}\left[\Phi\left(\xi_{1}, \xi_{2}, z ; q\right)\right], \\
(1-q) \zeta\left(\xi_{1}, \xi_{2} ; q\right)=c_{0} q \mathscr{N}_{2}\left[\Phi\left(\xi_{1}, \xi_{2}, z ; q\right), \zeta\left(\xi_{1}, \xi_{2} ; q\right)\right]
\end{gathered}
$$

and also the impermeable condition at the bottom

$$
\lim _{z \rightarrow-\infty} \frac{\partial \Phi}{\partial z}=0
$$

where $\mathscr{N}_{1}$ and $\mathscr{N}_{2}$ are two nonlinear operators defined by (2.11) and (2.12), $c_{0} \neq 0$ is the so-called 'convergence-control parameter' without physical meanings, respectively.

When $q=0$, the two boundary conditions (A 2) and (A 3) are automatically satisfied, thus we have the solution

$$
\Phi\left(\xi_{1}, \xi_{2}, z ; 0\right)=\varphi_{0}\left(\xi_{1}, \xi_{2}, z\right), \quad \zeta\left(\xi_{1}, \xi_{2} ; 0\right)=0
$$

since $\mathscr{L}[0]=0$ and the initial guess $\varphi_{0}\left(\xi_{1}, \xi_{2}, z\right)$ satisfies the Laplace equation (2.10) and the bottom boundary condition (2.13). When $q=1$, since $c_{0} \neq 0$, the zerothorder deformation equations (A 1)-(A 4) are equivalent to the original equations (2.10)(2.13), so that we have

$$
\Phi\left(\xi_{1}, \xi_{2}, z ; 1\right)=\varphi\left(\xi_{1}, \xi_{2}, z\right), \quad \zeta\left(\xi_{1}, \xi_{2} ; 1\right)=\eta\left(\xi_{1}, \xi_{2}\right) .
$$

Therefore, as $q$ increases from 0 to $1, \Phi\left(\xi_{1}, \xi_{2}, z ; q\right)$ varies from the initial guess $\varphi_{0}$ to the unknown velocity potential $\varphi\left(\xi_{1}, \xi_{2}, z\right)$, so does $\zeta\left(\xi_{1}, \xi_{2} ; q\right)$ from $z=0$ to the unknown wave elevation $\eta\left(\xi_{1}, \xi_{2}\right)$, respectively. Note that, in the frame of the HAM, we have great freedom to choose the auxiliary linear operator $\mathscr{L}$, the initial guess 
$\varphi_{0}\left(\xi_{1}, \xi_{2}, z\right)$ and the convergence-control parameter $c_{0}$. Assume that all of them are so properly chosen that both of $\Phi\left(\xi_{1}, \xi_{2}, z ; q\right)$ and $\zeta\left(\xi_{1}, \xi_{2} ; q\right)$ vary continuously and in addition that they are analytic with respect to the embedding parameter $q \in[0,1]$. Then, we have the Maclaurin series with respect to $q$ :

$$
\begin{gathered}
\Phi\left(\xi_{1}, \xi_{2}, z ; q\right)=\varphi_{0}\left(\xi_{1}, \xi_{2}, z\right)+\sum_{m=1}^{+\infty} \varphi_{m}\left(\xi_{1}, \xi_{2}, z\right) q^{m} \\
\zeta\left(\xi_{1}, \xi_{2} ; q\right)=\sum_{m=1}^{+\infty} \eta_{m}\left(\xi_{1}, \xi_{2}\right) q^{m}
\end{gathered}
$$

where

$$
\varphi_{m}\left(\xi_{1}, \xi_{2}, z\right)=\left.\frac{1}{m !} \frac{\partial^{m} \Phi\left(\xi_{1}, \xi_{2}, z ; q\right)}{\partial q^{m}}\right|_{q=0}, \quad \eta_{m}\left(\xi_{1}, \xi_{2}\right)=\left.\frac{1}{m !} \frac{\partial^{m} \zeta\left(\xi_{1}, \xi_{2} ; q\right)}{\partial q^{m}}\right|_{q=0} .(\mathrm{A} 9 a, b)
$$

Furthermore, assuming that the auxiliary linear operator $\mathscr{L}$ and especially the convergence-control parameter $c_{0}$ are so properly chosen that the above two Maclaurin series (A 7) and (A 8) are convergent at $q=1$, we have according to (A 6) the homotopy-series solution

$$
\begin{gathered}
\varphi\left(\xi_{1}, \xi_{2}, z\right)=\varphi_{0}\left(\xi_{1}, \xi_{2}, z\right)+\sum_{m=1}^{+\infty} \varphi_{m}\left(\xi_{1}, \xi_{2}, z\right), \\
\eta\left(\xi_{1}, \xi_{2}\right)=\sum_{m=1}^{+\infty} \eta_{m}\left(\xi_{1}, \xi_{2}\right),
\end{gathered}
$$

respectively.

Substituting the Maclaurin series (A 7) and (A 8) into the zeroth-order deformation equations (A 1)-(A 4) and then equating the like-power of $q$, we have the so-called high-order deformation equation

$$
\sum_{i=1}^{2} \sum_{j=1}^{2} \boldsymbol{k}_{i} \cdot \boldsymbol{k}_{j} \frac{\partial^{2} \varphi_{m}}{\partial \xi_{i} \partial \xi_{j}}+\frac{\partial^{2} \varphi_{m}}{\partial z^{2}}=0, \quad-\infty<z<0,
$$

subject to the linear boundary condition

$$
\mathscr{L}\left[\varphi_{m}\right]=c_{0} \Delta_{m-1}^{\phi}+\chi_{m} S_{m-1}-\bar{S}_{m}, \quad \text { on } z=0,
$$

and the bed condition

$$
\frac{\partial \varphi_{m}}{\partial z}=0, \quad \text { as } z \rightarrow-\infty
$$

together with

$$
\eta_{m}=c_{0} \Delta_{m-1}^{\eta}+\chi_{m} \eta_{m-1}, \quad \text { on } z=0,
$$

where $\chi_{1}=0$ and $\chi_{m}=1$ for $m>1$, and all of the terms $\Delta_{m-1}^{\eta}, \Delta_{m-1}^{\phi}, S_{m-1}, \bar{S}_{m}$ on the right-hand side of (A 13) are determined by the known previous approximations $\eta_{j}$ and $\varphi_{j}(j=0,1,2, \ldots, m-1)$, whose definitions are exactly the same as those given by Liao (2011). For the sake of simplicity, we neglect their detailed definitions here. 
Note that $\varphi_{m}\left(\xi_{1}, \xi_{2}, z\right)$ is in the form

$$
\varphi_{m}\left(\xi_{1}, \xi_{2}, z\right)=\sum_{i=0}^{+\infty} \sum_{j=-\infty}^{+\infty} b_{m}^{i, j} \Psi_{i, j}\left(\xi_{1}, \xi_{2}, z\right)
$$

where

$$
\Psi_{m, n}\left(\xi_{1}, \xi_{2}, z\right)=\sin \left(m \xi_{1}+n \xi_{2}\right) \exp \left(\left|m \boldsymbol{k}_{1}+n \boldsymbol{k}_{2}\right| z\right)
$$

automatically satisfies the Laplace equation (A 12) and the bed condition (A 14). Note that it is straightforward to gain $\eta_{m}$ by means of (A 15). So, only the linear boundary condition (A 13) need be satisfied. Thus, we have a special solution

$$
\varphi_{m}^{*}=\mathscr{L}^{-1}\left[c_{0} \Delta_{m-1}^{\phi}+\chi_{m} S_{m-1}-\bar{S}_{m}\right], \quad \text { on } z=0,
$$

where $\mathscr{L}^{-1}$ is the inverse operator of the auxiliary linear operator $\mathscr{L}$.

Note that the high-order deformation equations (A 12)-(A 14) are linear. Thus, in the frame of the HAM, we transfer the original nonlinear problem into an infinite number of linear sub-problems. However, unlike perturbation methods, such kind of transform does not need the existence of any small/large physical parameters. More importantly, different from other approximation techniques, we now have great freedom to choose the convergence-control parameter $c_{0}$, which has no physical meanings but provides us a simple way to guarantee the convergence of solution series. In particular, the HAM also provides us great freedom to choose the auxiliary linear operator $\mathscr{L}$ : in fact, it is this kind of freedom that provides us a straightforward way to handle the nearly resonant waves, as described below.

When the resonance criterion (2.9) is nearly satisfied, we choose a generalized auxiliary linear operator $\mathscr{L}$ defined by (2.33) with the definition (2.34), which has the property

$$
\mathscr{L}\left[\Psi_{m, n}\left(\xi_{1}, \xi_{2}, z\right)\right]=\bar{\lambda}_{m, n} \Psi_{m, n}\left(\xi_{1}, \xi_{2}, z\right),
$$

where $\bar{\lambda}_{m, n}$ defined by (2.26) is the eigenvalue of the generalized auxiliary linear operator (2.33). Therefore, its inverse operator has the property

$$
\mathscr{L}^{-1}\left[\Psi_{m, n}\left(\xi_{1}, \xi_{2}, z\right)\right]=\frac{\Psi_{m, n}\left(\xi_{1}, \xi_{2}, z\right)}{\bar{\lambda}_{m, n}}, \quad \text { when } \bar{\lambda}_{m, n} \neq 0
$$

Using this property, it is easy to gain the special solution $\varphi_{m}^{*}$ by means of (A 18).

Note that the generalized auxiliary linear operator (2.33) always have three eigenvalues being zero:

$$
\bar{\lambda}_{1,0}=\bar{\lambda}_{0,1}=\bar{\lambda}_{2,-1}=0 \text {. }
$$

Therefore, $\Psi_{1,0}, \Psi_{0,1}$ and $\Psi_{2,-1}$ are three primary solutions. So, the common solution reads

$$
\varphi_{m}=\varphi_{m}^{*}+a_{m}^{1,0} \Psi_{1,0}+a_{m}^{0,1} \Psi_{0,1}+a_{m}^{2,-1} \Psi_{2,-1},
$$

where $\varphi_{m}^{*}$ is a special solution, and $a_{m}^{0,1}, a_{m}^{1,0}$ and $a_{m}^{2,-1}$ are three constants to be determined.

In the frame of the HAM, we have freedom to choose such an initial guess

$$
\varphi_{0}=a_{0}^{1,0} \Psi_{1,0}+a_{0}^{0,1} \Psi_{0,1}+a_{0}^{2,-1} \Psi_{2,-1}
$$


where $a_{0}^{0,1}, a_{0}^{1,0}$ and $a_{0}^{2,-1}$ are three constants, which are determined by enforcing the disappearance of the terms

$$
\sin \xi_{1}, \quad \sin \xi_{2}, \quad \sin \left(2 \xi_{1}-\xi_{2}\right)
$$

on the right-hand side of (A 13), since the corresponding eigenvalues $\bar{\lambda}_{1,0}, \bar{\lambda}_{0,1}$ and $\bar{\lambda}_{2,-1}$ are zero so that (A 20) is invalid. This provides us three coupled, nonlinear algebraic equations about the unknown constants $a_{0}^{0,1}, a_{0}^{0,1}$ and $a_{0}^{2,-1}$, which often have multiple solutions with real values, indicating multiple steady-state resonant waves, as pointed out by Liao (2011). In this way, we gain the initial guess $\varphi_{0}$, which further gives $\eta_{1}$ by means of (A 15). Then, it is straightforward to gain the special solution $\varphi_{1}^{*}$ by means of (A 18), and furthermore to have the common solution

$$
\varphi_{1}=\varphi_{1}^{*}+a_{1}^{1,0} \Psi_{1,0}+a_{1}^{0,1} \Psi_{0,1}+a_{1}^{2,-1} \Psi_{2,-1},
$$

where $a_{1}^{1,0}, a_{1}^{0,1}$ and $a_{1}^{2,-1}$ are unknown constants, which can be determined in a similar way. In this way, we can gain $\varphi_{m}$ and $\eta_{m}$ at high enough order of approximation, especially by means of computer algebra software such as Mathematica, Maple and so on.

Note that the right-hand side of (A 13) contains the convergence-control parameter $c_{0}$, which provides us a simple way to guarantee the convergence of the solution series. The optimal value of the convergence-control parameter $c_{0}$ is determined by the minimum of the sum of residual error square of the two free surface boundary conditions. For example, please refer to table 1.

Note that we have $\mu=2$ when the resonance criterion (2.9) is exactly satisfied. Therefore, the auxiliary linear operator (2.23) used by Liao (2011) for the exactly resonant waves is only a special case of the generalized auxiliary linear operator (2.33) for the nearly resonant waves. In addition, all mathematical formulae for the steadystate nearly resonant waves are the same as those given by Liao (2011) for the exactly resonant ones, only except the generalized auxiliary linear operator (2.33). So, the above-mentioned HAM-based approach is valid for both of the exactly resonant waves and nearly resonant waves. This indicates from the viewpoint of the HAM that exactly resonant waves have no fundamental difference from nearly resonant ones. The results given in $\S 3$ also confirm this viewpoint.

In general, no matter either the resonance criterion

$$
\boldsymbol{k}_{3}=m^{\prime} \boldsymbol{k}_{1}-n^{\prime} \boldsymbol{k}_{2}, \quad \omega_{3}=m^{\prime} \omega_{1}-n^{\prime} \omega_{2}
$$

is satisfied exactly or nearly, the above-mentioned HAM-based approach is valid, as long as we choose

$$
\mu=\frac{g\left|m^{\prime} \boldsymbol{k}_{1}+n^{\prime} \boldsymbol{k}_{2}\right|-\left(m^{\prime 2} \omega_{1}^{2}+n^{\prime 2} \omega_{2}^{2}\right)}{m^{\prime} n^{\prime} \omega_{1} \omega_{2}}
$$

in the generalized auxiliary linear operator (2.23), where $m^{\prime}$ and $n^{\prime}$ are integers.

\section{Appendix B. Approach based on the Zakharov's equation}

$\mathrm{Xu}$ et al. (2012) first gained the steady-state exactly resonant waves by means of the exact wave equations, and then confirmed their results by means of the Zakharov's equation, which is a simplified wave model for exactly resonant and nearly resonant 
waves with weak nonlinearity. Here, to verify the HAM-based approach described in $\S 2$ and the results of the case study reported in $\S 3$, we solve here the same case by means of the Zakharov's equation.

Let us consider a degenerate quartet in a linear near-resonance:

$$
\begin{gathered}
2 \boldsymbol{k}_{1}-\boldsymbol{k}_{2}-\boldsymbol{k}_{3}=0, \\
2 \omega_{1}-\omega_{2}-\omega_{3}=\Delta \omega, \\
2 \sigma_{1}-\sigma_{2}-\sigma_{3}=0,
\end{gathered}
$$

which give

$$
\epsilon_{3}=\frac{2 \epsilon_{1} \omega_{1}-\epsilon_{2} \omega_{2}}{2 \omega_{1}-\omega_{2}-\Delta \omega}
$$

where

$$
\epsilon_{i}=\frac{\sigma_{i}}{\omega_{i}}, \quad i=1,2,3
$$

For the same case (3.1), we take

$$
\epsilon_{1}=\epsilon_{2}=\epsilon \text {. }
$$

Substituting

$$
B(\boldsymbol{k}, t)=B_{1}(t) \delta\left(\boldsymbol{k}-\boldsymbol{k}_{1}\right)+B_{2}(t) \delta\left(\boldsymbol{k}-\boldsymbol{k}_{2}\right)+B_{3}(t) \delta\left(\boldsymbol{k}-\boldsymbol{k}_{3}\right)
$$

into Zakharov's equation

$$
\mathrm{i} \frac{\mathrm{d} B}{\mathrm{~d} t}=\iiint_{-\infty}^{+\infty} T_{1123} B_{1}^{*} B_{2} B_{3} \delta_{1+1-2-3} \mathrm{e}^{\mathrm{i}\left(2 \omega_{1}-\omega_{2}-\omega_{3}\right) t} \mathrm{~d} \boldsymbol{k}_{1} \mathrm{~d} \boldsymbol{k}_{2} \mathrm{~d} \boldsymbol{k}_{3}
$$

and using (B 1), we have

$$
\begin{aligned}
\mathrm{i} \frac{\mathrm{d} B_{1}}{\mathrm{~d} t} & =\left(T_{1111}\left|B_{1}\right|^{2}+2 T_{1212}\left|B_{2}\right|^{2}+2 T_{1313}\left|B_{3}\right|^{2}\right) B_{1}+2 T_{1123} \mathrm{e}^{\mathrm{i} \Delta \omega t} B_{1}^{*} B_{2} B_{3}, \\
\mathrm{i} \frac{\mathrm{d} B_{2}}{\mathrm{~d} t} & =\left(2 T_{2121}\left|B_{1}\right|^{2}+T_{2222}\left|B_{2}\right|^{2}+2 T_{2323}\left|B_{3}\right|^{2}\right) B_{2}+T_{2311} \mathrm{e}^{-\mathrm{i} \Delta \omega t} B_{3}^{*} B_{1}^{2} \\
\mathrm{i} \frac{\mathrm{d} B_{3}}{\mathrm{~d} t} & =\left(2 T_{3131}\left|B_{1}\right|^{2}+2 T_{3232}\left|B_{2}\right|^{2}+T_{3333}\left|B_{3}\right|^{2}\right) B_{3}+T_{3211} \mathrm{e}^{-\mathrm{i} \Delta \omega t} B_{2}^{*} B_{1}^{2}
\end{aligned}
$$

where $T_{i j k l}$ is a kernel that can be found in the textbook of Mei et al. (2005). For steady-state solutions, it holds

$$
B_{j}(t)=\beta_{j} \mathrm{e}^{-\mathrm{i} \Omega_{j} t}, \quad j=1,2,3,
$$

where $\beta_{j}=\left|\beta_{j}\right| \mathrm{e}^{\mathrm{i} a r g \beta_{j}}$ with $\left|\beta_{j}\right|$, arg $\beta_{j}$ being unknown real constants, and

$$
\Omega_{j}=\left(\epsilon_{j}-1\right) \omega_{j}
$$

Substituting (B 10) into (B 9a-c) and using (B 3), we have the nonlinear algebraic equations

$$
\Omega_{1}=T_{1111}\left|\beta_{1}\right|^{2}+2 T_{1212}\left|\beta_{2}\right|^{2}+2 T_{1313}\left|\beta_{3}\right|^{2}+2 T_{1123} \beta_{1}^{-1} \beta_{1}^{*} \beta_{2} \beta_{3},
$$




$$
\begin{aligned}
& \Omega_{2}=2 T_{2121}\left|\beta_{1}\right|^{2}+T_{2222}\left|\beta_{2}\right|^{2}+2 T_{2323}\left|\beta_{3}\right|^{2}+T_{2311} \beta_{1}^{2} \beta_{2}^{-1} \beta_{3}^{*}, \\
& \Omega_{3}=2 T_{3131}\left|\beta_{1}\right|^{2}+2 T_{3232}\left|\beta_{2}\right|^{2}+T_{3333}\left|\beta_{3}\right|^{2}+T_{2311} \beta_{1}^{2} \beta_{2}^{*} \beta_{3}^{-1} .
\end{aligned}
$$

And for steady-state quartets with time-independent spectrum, one must set

$$
2 \arg \beta_{1}-\arg \beta_{2}-\arg \beta_{3}=n \pi, \quad(n=0,1) .
$$

Note that the steady-state free-surface elevation for the quartet is

$$
\eta=\sum_{j=1,2,3} a_{j} \cos \left[\boldsymbol{k}_{j} \cdot \boldsymbol{x}-\left(\omega_{j}+\Omega_{j}\right) t+\arg \beta_{j}\right],
$$

where the wave amplitudes $a_{j}$ are related to $\beta_{j}$ by

$$
a_{j}=\left(\frac{\omega_{j}}{2 g}\right)^{1 / 2} \frac{\left|\beta_{j}\right|}{\pi}, \quad j=1,2,3 .
$$

Thus, as soon as we get the solution $\left|\beta_{1}\right|,\left|\beta_{2}\right|,\left|\beta_{3}\right|$ from the algebraic equations (B 12)-(B 14) with (B 15) for given $\omega_{j}$ and $\epsilon_{j}, j=1,2,3$, the corresponding wave amplitudes are determined by (B 17).

\section{REFERENCES}

BenNey, D. J. 1962 Non-linear gravity wave interactions. J. Fluid Mech. 14 (4), 577-584.

Bretherton, F. P. 1964 Resonant interactions between waves. The case of discrete oscillations. J. Fluid Mech. 20 (3), 457-479.

Concus, P. 1962 Standing capillary-gravity waves of finite amplitude. J. Fluid Mech. 14, 568-576.

Craig, W. \& Nicholls, D. P. 2000 Traveling two and three dimensional capillary gravity water waves. SIAM J. Math. Anal. 32 (2), 323-359.

Craig, W. \& Nicholls, D. P. 2002 Traveling gravity water waves in two and three dimensions. Eur. J. Mech. (B/Fluids) 21 (6), 615-641.

Dubrovin, B. A., Flickinger, R. \& Segur, H. 1997 Three-phase solutions of the KadomtsevPetviashvili equation. Stud. Appl. Maths 99, 137-203.

HAMMACK, J. L. \& Henderson, D. M. 1993 Resonant interactions among surface water waves. Annu. Rev. Fluid Mech. 25 (1), 55-97.

Hammack, J. L., Henderson, D. M. \& Segur, Harvey 2005 Progressive waves with persistent two-dimensional surface patterns in deep water. J. Fluid Mech. 532, 1-52.

Hammack, J., Scheffner, N. \& Segur, H. 1989 Two-dimensional periodic waves in shallow water. J. Fluid Mech. 209, 567-589.

Iooss, G. \& Plotnikov, P. 2009 Existence of a directional stokes drift in asymmetrical threedimensional travelling gravity waves. C. R. Méc. 337, 633-638.

Iooss, G. \& Plotnikov, P. 2011 Asymmetrical three-dimensional travelling gravity waves. Arch. Rat. Mech. Anal. 200, 789-880.

LiAO, S. J. 1992 Proposed homotopy analysis techniques for the solution of nonlinear problems. $\mathrm{PhD}$ thesis, Shanghai Jiao Tong University.

LIAO, S. J. 1997 An approximate solution technique which does not depend upon small parameters (2): an application in fluid mechanics. Intl J. Non-Linear Mech. 32, 815-822.

Liao, S. J. 2003 Beyond Perturbation - An Introduction to Homotopy Analysis Method. Chapman \& Hall/CRC.

LiaO, S. J. 2004 On the homotopy analysis method for nonlinear problems. Appl. Maths Comput. 147, 499-513. 
LiaO, S. J. 2011 On the homotopy multiple-variable method and its applications in the interactions of nonlinear gravity waves. Commun. Nonlinear Sci. Numer. Simul. 16 (3), 1274-1303.

Liao, S. J. 2012 Homotopy Analysis Method in Nonlinear Differential Equations. Springer \& Higher Education Press.

LiAO, S. J. 2014 Do peaked solitary water waves indeed exist? Commun. Nonlinear Sci. Numer. Simul. 19, 1792-1821.

LIAO, S. J. \& TAN, Y. 2007 A general approach to obtain series solutions of nonlinear differential equations. Stud. Appl. Maths 119, 297-354.

LiU, Z. \& LiaO, S. J. 2014 Steady-state resonance of multiple wave interactions in deep water. J. Fluid Mech. 742, 664-700.

LiU, Z., Xu, D. L., Li, J., Peng, T., Alsaedi, A. \& Liao, S. J. 2015 On the existence of steady-state resonant waves in experiment. J. Fluid Mech. 763, 1-23.

Longuet-Higgins, M. S. 1962 Resonant interactions between two trains of gravity waves. J. Fluid Mech. 12 (3), 321-332.

Longuet-Higgins, M. S. \& SMith, N. D. 1966 An experiment on third-order resonant wave interactions. J. Fluid Mech. 25 (3), 417-435.

Madsen, P. A. \& Fuhrman, D. R. 2012 Third-order theory for multi-directional irregular waves. J. Fluid Mech. 698, 304-334.

Mei, C. C., Stiassnie, M. \& Yue, D. K. P. 2005 Theory and Applications of Ocean Surface Waves: Nonlinear Aspects. World Scientific.

Meiron, D. I., Saffman, P. G. \& Yuen, H. C. 1982 Calculation of steady three-dimensional deep-water waves. J. Fluid Mech. 124, 109-121.

Milewski, P. A. \& Keller, J. B. 1996 Three-dimensional water waves. Stud. Appl. Maths 37, 149-166.

NichOLLS, D. P. 1998 Traveling water waves: spectral continuation methods with parallel implementation. J. Comput. Phys. 142, 224-240.

Nicholls, D. P. \& Reitich, F. 2006 Stable, high-order computation of traveling water waves in three dimensions. Eur. J. Mech. (B/Fluids) 25 (4), 406-424.

Onorato, M., Osborne, A. R., Janssen, P. \& Resio, D. 2009 Four-wave resonant interactions in the classical quadratic boussinesq equations. J. Fluid Mech. 618, 263-277.

PhILliPS, O. M. 1960 On the dynamics of unsteady gravity waves of finite amplitude. J. Fluid Mech. 9, 193-217.

PoInCARÉ, H. 1892 Les méthodes nouvelles de la mécanique céleste. Gauthier-Villars.

STIASSNIE, M. \& SHEMER, L. 1984 On modifications of the Zakharov equation for surface gravity waves. J. Fluid Mech. 143, 47-67.

Su, M. A. 1982 Three-dimensional deep-water waves. Part 1 - experimental measurement of skew and symmetric wave patterns. J. Fluid Mech. 124, 73-108.

Su, M. A., Bergin, M., MARler, P. \& MYrick, R. 1982 Experiments on nonlinear instabilities and evolution of steep gravity-wave trains. J. Fluid Mech. 124, 45-72.

Vajravelu, K. \& Van Gorder, R. A. 2013 Nonlinear Flow Phenomena and Homotopy Analysis. Springer \& Higher Education Press.

XU, D. L., LiN, Z. L. \& LiAO, S. J. 2015 Equilibrium states of class-I Bragg resonant wave system. Eur. J. Mech. (B/Fluids) 50, 38-51.

Xu, D. L., Lin, Z. L., LiaO, S. J. \& Stiassnie, M. 2012 On the steady-state fully resonant progressive waves in water of finite depth. J. Fluid Mech. 710, 379-418.

Yoccoz, J. C. 1992 An introduction to small divisors problems. In From Number Theory to Physics, chap. 14 , Springer. 